NASA Technical Memorandum 87165

\title{
Effect of Interference Fits on Roller Bearing Fatigue Life
}

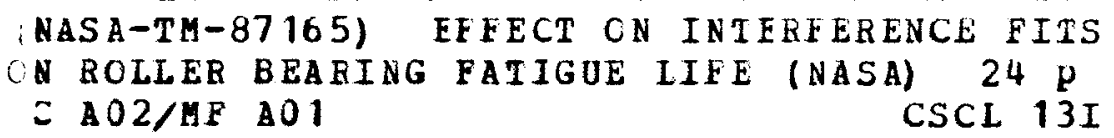

Harold H. Coe and Erwin V. Zaretsky

\section{Lewis Research Center}

Cleveland, Ohio

January 1986

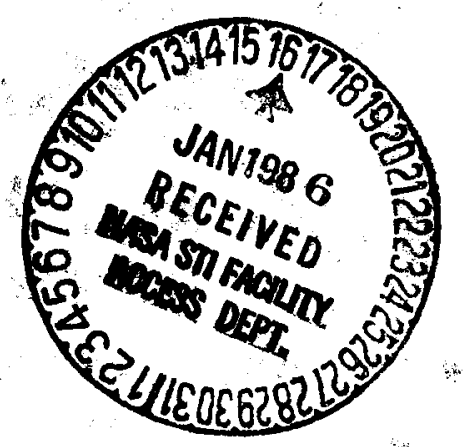




\title{
EFFECT OF INTERFERENCE FITS ON ROLLER BEARING FATIGUE LIFE
}

\author{
Harold H. Coe and Erwin V. Zaretsky \\ National Aeronautics and Space Administration \\ Lewis Research Center \\ Cleveland, ohio 44135
}

\begin{abstract}
SUMMARY
An analysis was performed to determine the effects of inner-ring speed and press fits on roller bearing fatigue life. The effects of the resultant hoop and radial stresses on the principal stresses were considered. The maximum shear stresses below the Hertzian contact were determined for different conditions of inner ring speed and load, and were applied to a conventional roller bearing life analysis. The effect of mean stress was determined using a Goodman diagram approach. Hoop stresses caused by press fits and centrifugal force can reduce bearing 11 fe by as much as 90 percent. Use of a Goodman diagram predicted life reduction of 20 to 30 percent. The depth of the maximum shear stress remained virtually unchanged.
\end{abstract}

NOMENCLATURE

A defined by equation (48), N/M2 (1b/in. $\left.{ }^{2}\right)$

B defined by equation (25) dimensionless

b semiwidth of contact area (see fig. 1), M (in.)

c stress-1ife exponent

E modulus of elasticity, $N / M^{2}\left(1 b / i n .{ }^{2}\right)$

$E^{\prime}$ defined by equation (13), $M^{2} / N($ in. $2 / 1 b)$

e Wefbull slope (exponent)

G defined by equation (30) dimensionless

g gravitational constant, $M / \sec ^{2}\left(\mathrm{in} / \mathrm{sec}^{2}\right)$

h exponent

K defined by equation (30), N-sec/M4 $\left(1 \mathrm{~b}-\mathrm{sec} / 1 \mathrm{n}^{4}\right)$

$K_{1}$ constant relating press fit to pressure $\left(=2 / E\left(1-B^{2}\right)\right.$ ) (for a solid shaft)

$K_{2}$ defined by equation (45) dimensionless

K3 material constant

$\mathrm{L}$ contact fatigue $11 \mathrm{fe}, \mathrm{hr}$ 
LR Iife ratio, defined by equation (52)

$l$ length of contact area (fig. 1), $M($ in.)

m defined by equation (48), $N / M^{2}\left(1 \mathrm{~b} / \mathrm{in} .{ }^{2}\right)$

$P_{i}$ pressure on $r_{1}$, due to press fit, $N / M^{2}\left(1 b / i n .{ }^{2}\right)$

$P_{0}$, pressure on $r_{0}, N / M^{2}\left(1 b / i n .{ }^{2}\right)$

$P_{0}^{\prime}$ roller load, $N(1 b)$

$R$ radius of curvature, $M$ (in.)

$R^{\prime} \quad$ ratio, defined by equation (44) dimensionless

$R_{r}$ radius of roller (fig. 3 ), $M($ in.)

$r$ radius to element (fig. 3 ), $M($ in.)

$r_{1}$ ring inner radius (fig. 3$), M($ in.)

$r_{0} \quad$ ring outer radius (fig. 3 ), $M($ in.)

$S_{\max }$ Hertz stress at center of contact, $N / M^{2}\left(1 b / 1 n .{ }^{2}\right)$

Su ultimate strength of material, $\mathrm{N} / \mathrm{m}^{2}\left(1 \mathrm{~b} / \mathrm{in} .{ }^{2}\right)$

Sy principal stress in the Y-direction (eq. (34)), N/M2 (lb/in. ${ }^{2}$ )

$S_{Z}$ principal stress in the Z-direction (eq. (33)), $N / M^{2}\left(1 \mathrm{~b} /\right.$ in. ${ }^{2}$ )

$t \quad$ simplifying term $\left(=\sqrt{1+u^{2}}\right)$

$u$ dimensionless depth below surface $(=2 / b)$

$v \quad$ stressed volume, $M^{3}\left(\right.$ in. $\left.{ }^{3}\right)$

$x$ axis perpendicular to rolling direction

$x_{x} \quad$ principal stress, $X$-direction, Hertz loading only (eq. (4)), N/M2 $(1 b /$ in. 2$)$

$Y \quad$ axis in direction of rolling

Yy principal stress, Y-direction, Hertz loading only (eq. (5)), N/M2 $(1 \mathrm{~b} / \mathrm{in} .2)$

y defined by eq. (25) dimensionless

$y_{\max }$ value of $y$ at $\tau_{\max }$ dimensioniess

Z distance below Hertz contact surface (fig. 1), $M$ (in.) or axis in direction of Hertzian loading 
Zo value of depth to maximum shearing stress, $M$ (in.)

$z_{Z}$ principal stress, Z-direction, Hertz loading only (eq. (6)), N/M2 $(1 \mathrm{~b} / 1 \mathrm{n.2})$

$\gamma$ density of material, $N / M^{3}\left(1 b / i n .{ }^{3}\right)$

$\Delta$ press fit on radius, $M($ in.)

$\delta$ Poisson's ratio (also v) dimensioniess

$\theta$ defined by eq. (10), N/M2 (in.2/1b)

$\Lambda$ defined by eq. (15), $m^{3} / N($ in. $3 / 1 b)$

$v$ Poisson's ratio (also $\delta$ ) dimensioniess

$\pi \quad P_{1}(=3.14159)$

$\Sigma_{\rho} \quad$ curvature $\operatorname{sum}(=1 / R a+1 / R b), 1 / M\left(1 / 1 n_{.}\right)$

o stress, $N / M^{2}\left(1 b / i n .{ }^{2}\right)$

$\tau$ maximum shear stress, $N / M^{2}\left(1 \mathrm{~b} /\right.$ in. $\left.{ }^{2}\right)$

$\tau$ a amplitude of $T, N / M^{2}\left(1 b / i n .{ }^{2}\right)$

Te effective maximum shear stress, $N / M^{2}\left(1 \mathrm{~b} / 1 \mathrm{n} .{ }^{2}\right)$

$\tau_{m}$ mean value of $\tau, N / M^{2}\left(1 \mathrm{~b} / \mathrm{in} .{ }^{2}\right)$

$\tau_{\max }$ maximum value of $T, N / M^{2}\left(1 b / i n .{ }^{2}\right)$

$\omega$ inner ring speed, $\mathrm{rad} / \mathrm{sec}$

\section{Subscripts}

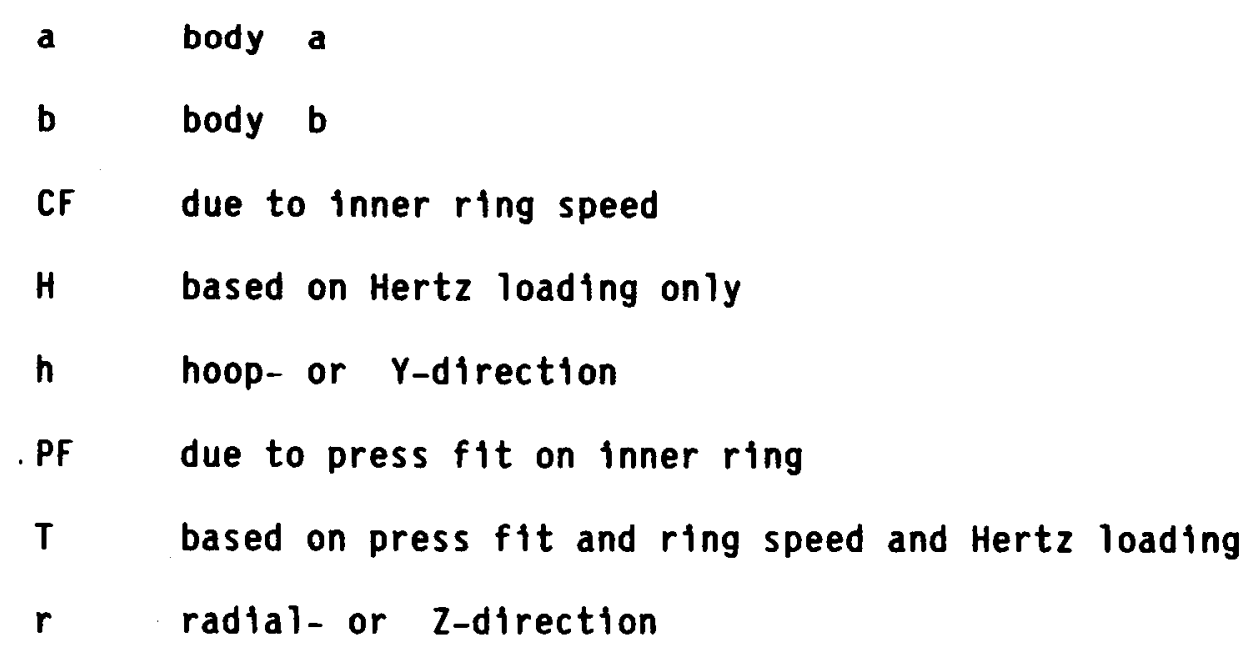




\section{INTRODUCTION}

With advancements in turbomachinery, rolling-element bearing operating speeds and temperatures have been steadily increasing. Because of centrifugal loading on the bearing inner ring-shaft interface, and differential temperatures, the inner ring expands or grows relative to the shaft. This can result, even in a highly loaded bearing application, in the inner ring "walking" around the shaft. The most benign effect of this phenomenon is replacement of the bearing at overhaul and plating and regrinding of the shaft at the bearing position due to wear. However, in the most extreme cases, the wear of the shaft can either act as a stress raiser to cause fatigue or fracture of the shaft or in the case of hollow shafts actually wear through the shaft. In these cases, catastrophic or secondary damage can occur.

In order to prevent motion of the inner ring around a shaft, designers have been specifying very tight interference fit between the inner $r i n g$ and shaft where it is not practical to provide for a keyway or locknut arrangement. The interference fit is usualiy based upon the anticipated growth of the shaft and bearing under the most severe operating conditions. These conditions sometimes only exist for short time perlods in the machine's operating cycle. Nevertheless, it is a very important design consideration for both safety of operation as well as maintainability. In recent years it has been noticed by some engineers that bearings which have higher than usual interference or press fits may have field lives which are shorter than those which were anticipated or calculated. The fallure mechanism is usually classical rolling-element (subsurface) fatigue. There has been no public documentation of the phenomenon.

Residual compressive stresses near the surface of a roling element increases the rolling-element fatigue life of the surface under rolling contact loading (refs. 1 to 4 ). These compressive residual stresses can be deve1oped for a number of processing operations such as grinding and shot peening (ref. 4). Tensile residual stresses while not common can negatively affect fatigue life. These tensile residual stresses would be analogous to those hoop tensile stresses which may be induced due to press fits and ring growth due to thermal and centrifugal effects. If residual stress can affect the fatigue Iife, then these other stresses which can be present may also alter the critical subsurface shearing stress and affect rolling-element fatigue life of the bearing inner race.

Czyzewski (ref. 5) first postulated that tensile stresses in a cylindrical race imposed on a lubricated Hertzian contact would affect shearing stresses and, hence, rolling-element fatigue life. He performed an analysis and rolling-element fatigue tests of $45-\mathrm{mm}$ bore roller bearing inner-races subjected to mechanically induced tensile stress. Maximum Hertzian stress was approximately $700 \mathrm{MN} / \mathrm{m}^{2}\left(102 \times 10^{3} \mathrm{psi}\right)$. There is a suggestion of an inverse ninth power stress-life relation. At a hoop tensile stress of $80 \mathrm{mN} / \mathrm{m}^{2}$ ( $12 \times 10^{3}$ psi) fallure appears to be by a surface fatigue spall accompanied by ring fracture. At the lower hoop stresses, the fallure mode was by classical rolling-element fatigue.

The research reported herein was undertaken to investigate the effects of inner-ring press fits and centrifugal force on roller bearing life. The objectives were (a) develop an equation to account for the effect of hoop stresses 
on maximum shearing stress of a Hertzian contact and (b) determine the magnitude of the effects of press fit and centrifugal force on roller bearing life.

\section{ANALYSIS}

Current evaluations of rolling-element fatigue life are based on either the orthogonal shear stress or the maximum shearing stress (ref. 6) which occur in a zone under the rolling-element contact surfaces. These shearing stresses are a function of the contact (Hertz) stress due to two bodies in contact. The Hertzian stress, in turn is a function of load, geometry, and material physical properties of the rolling-element bodies. Jones (ref. 7 ), based on the work of Thomas and Hoersch (ref. 8) shows the principal stresses (fig. 1) due to a Hertzian loading to be:

$$
\begin{aligned}
& \frac{\Delta X_{X}}{b}=-2 \delta\left[\sqrt{1+\left(\frac{Z}{b}\right)^{2}}-\frac{Z}{b}\right] \\
& \frac{\Delta Y_{Y}}{b}=\frac{-\left[\sqrt{1+\left(\frac{Z}{b}\right)^{2}}-\frac{Z}{b}\right]^{2}}{\sqrt{1+\left(\frac{Z}{b}\right)^{2}}} \\
& \frac{\Delta Z_{Z}}{b}=-\frac{1}{\sqrt{1+\left(\frac{Z}{b}\right)^{2}}}
\end{aligned}
$$

By letting $u=Z / b$ and $t=\sqrt{1+u^{2}}$, these equations become

$$
\begin{gathered}
\frac{\Delta X_{X}}{b}=-2 \delta(t-u) \\
\frac{\Lambda Y_{Y}}{b}=\frac{-(t-u)^{2}}{t} \\
\frac{\Lambda Z Z}{b}=-\frac{1}{t}
\end{gathered}
$$

For roller bearings, the maximum shear stress is determined by the principal stresses in the $Z$ (direction of Hertzian loading) and the $Y$ (direction of rolling) direction. Thus

$$
\tau=\frac{1}{2}\left(Z_{Z}-Y_{Y}\right)
$$

So, for Hertzian loading only, substituting from Eqs. (2) and (3) into equation (7) gives

$$
\tau=\frac{b}{\Lambda}\left(t-u-\frac{l}{t}\right)
$$


But, from reference 7 ,

$$
\begin{aligned}
& b^{2}=\frac{P_{0}^{\prime}\left(\theta_{a}+\theta_{b}\right)}{\pi l} \\
& \theta_{a}=\frac{4\left(1-\delta_{a}^{2}\right)}{E_{a}} \\
& \theta_{b}=\frac{4\left(1-\delta_{b}^{2}\right)}{E_{b}} \\
& \Lambda=\left[\frac{1-\delta_{a}^{2}}{E_{a}}+\frac{1-\delta_{b}^{2}}{E_{b}}\right] \frac{2}{\Sigma_{p}} \\
& S_{\text {max }}=\frac{2 P_{0}^{\prime}}{\pi l b} \\
& E^{\prime}=\frac{1-\delta_{a}^{2}}{E_{a}}+\frac{1-\delta_{b}^{2}}{E_{b}} \\
& \theta_{a}+\theta_{b}=4 E^{\prime} \\
& \Lambda=\frac{2 E^{\prime}}{\Sigma \rho}
\end{aligned}
$$

Equation (9) can now be written

or

$$
\begin{gathered}
b=\left(\frac{2 P_{0}^{\prime}}{\pi \ell b}\right)\left(\frac{2 E^{\prime}}{\sqrt{p}}\right) \\
b=S_{\max } \Lambda
\end{gathered}
$$

Substituting equation (17) into equation (8) gives

$$
\tau=S_{\max }\left(t-u-\frac{1}{t}\right)
$$

Thus, the shear stress, made nondimensional by the maximum Hertz stress, is a direct function of $u$, the depth below the surface, as shown in figure 2. A maximum value of the maximum shear stress occurs at a depth (u) of approximately 0.78 . By taking the derivative of equation (18) with respect to $u$ and setting the result equal to zero, one obtains

$$
\frac{d \tau}{d u}=S_{\max }\left(\frac{u}{t}+\frac{u}{t^{3}}-1\right)=0
$$


The solution to equation (19) is $u=0.786152$, and the corresponding equation for the maximum shear stress becomes

$$
\tau_{\max }=-0.30028 S_{\max }
$$

Equations (18) and (20) account for the subsurface stresses due only to the Hertzian contact and do not account for the effects of press fitting an inner ring on a shaft or for the effects of inner-ring speed. These effects can be accounted for, using the method of superposition, by determining the values of subsurface stress in the $Z$ and $Y$ direction as a function of depth below the surface to correspond with equations (5) and (6).

\section{Stress Due to Press Fit}

From reference 9 we can write, for the hoop stress

$$
\left(\sigma_{h}\right)_{P F}=\frac{1}{r_{0}^{2}-r_{1}^{2}}\left[r_{1}^{2} p_{1}-r_{0}^{2} p_{0}+\left(\frac{r_{i} r_{0}}{r}\right)^{2}\left(p_{1}-p_{0}\right)\right]
$$

Note that $P_{0}=0$ and that $P_{j}$ is due to a press fit of the ring on a shaft, that is,

$$
P_{1}=\frac{\Delta}{r_{1} K_{1}}
$$

Equation (21) can be written

$$
\left(\sigma_{h}\right)_{P F}=\frac{P_{i} r_{1}^{2}}{r_{0}^{2}-r_{1}^{2}}\left[1+\left(\frac{r_{0}}{r}\right)^{2}\right]
$$

Similarly, we can write, for the radial stress

$$
\left(\sigma_{r}\right)_{P F}=\frac{P_{1} r_{i}^{2}}{r_{0}^{2}-r_{i}^{2}}\left[1-\left(\frac{r_{0}}{r}\right)^{2}\right]
$$

If we let

$$
\frac{r_{1}}{r_{0}}=B \text { and } \frac{r}{r_{0}}=y
$$

Equations (23) and (24) become

$$
\left(\sigma_{h}\right)_{P F}=\frac{P_{i} B^{2}}{1-B^{2}}\left(1+\frac{1}{y^{2}}\right)
$$




$$
\left(\sigma_{r}\right)_{P F}=\frac{P_{f} B^{2}}{1-B^{2}}\left(1-\frac{1}{y^{2}}\right)
$$

\section{Stresses Due to Inner Ring Speed}

From reference 9 we can write, for the hoop stress,

$$
\left(\sigma_{h}\right)_{C F}=\frac{3-2 v}{8(1-v)}\left(\frac{r \omega^{2}}{g}\right)\left[r_{0}^{2}+r_{i}^{2}+\left(\frac{r_{0} r_{i}}{r}\right)^{2}-r^{2}\left(\frac{1-2 v}{3-2 v}\right)\right]
$$

Similarly, for the radial stress

let

$$
\begin{gathered}
\left(\sigma_{r}\right)_{C F}=\frac{3-2 v}{8(1-v)}\left(\frac{\gamma \omega^{2}}{g}\right)\left[r_{0}^{2}-r_{1}^{2}-r^{2}-\left(\frac{r_{0} r_{1}}{r}\right)^{2}\right] \\
K=\left(\frac{3-2 v}{8(1-v)}\right)\left(\frac{Y}{g}\right), \quad G=\frac{1-2 v}{3-2 v}
\end{gathered}
$$

and using equation (25), equations (28) and (29) become

$$
\begin{aligned}
& \left(\sigma_{h}\right)_{C F}=K \omega^{2} r_{0}^{2}\left[1+B^{2}+\frac{B^{2}}{y^{2}}-G y^{2}\right] \\
& \left(\sigma_{r}\right)_{C F}=K \omega^{2} r_{0}^{2}\left[1-B^{2}-\frac{B^{2}}{y^{2}}-y^{2}\right]
\end{aligned}
$$

\section{Combined Stresses}

By the method of superposition, the principal stresses in the $Z$ and $Y$ directions become

$$
\begin{aligned}
& s_{Z}=Z_{Z}+\left(\sigma_{r}\right)_{P F}+\left(\sigma_{r}\right)_{C F} \\
& s_{y}=Y_{Y}+\left(\sigma_{h}\right)_{P F}+\left(\sigma_{h}\right)_{C F}
\end{aligned}
$$

and the maximum shear stress is

$$
\tau=\frac{1}{2}\left(S_{z}-S_{y}\right)
$$

To utilize these equations, however, one must determine the relation between $u$ and $y$ so that the stresses can all be determined at the same location. 
From geometry, (figs. (1) and (3))

$$
r=r_{0}-z=r_{0}-u b
$$

or

$$
\frac{r}{r_{0}}=1-\frac{u b}{r_{0}}=y
$$

and

$$
u=\frac{r_{0}}{b}(1-y)
$$

Substituting equation (17) gives

$$
u=\frac{r_{0}}{S_{\max } \Lambda}(1-y)
$$

From figure (3)

$$
\Sigma_{\rho}=\frac{1}{r_{0}}+\frac{1}{R_{r}}=\frac{R_{r}+r_{0}}{r_{0} R_{r}}
$$

Substituting equation (40) into equation (15) gives

$$
\Lambda=\frac{2 E^{\prime} r_{0} R_{r}}{r_{0}+R_{r}}
$$

Using equation (41) in equation (39) lets us write

$$
u=\frac{\left(\frac{r_{0}}{R_{r}}+1\right)}{2 S_{\max } E^{\prime}}(1-y)
$$

or

$$
u=\frac{R^{\prime}+1}{2 S_{\max } E^{\prime}}(1-y)
$$

where

$$
R^{\prime}=\frac{r_{0}}{R_{r}}
$$

For simplicity, let

then

$$
K_{2}=\frac{R^{\prime}+1}{2 S_{\max } E^{\prime}}
$$

$$
u=k_{2}(1-y)
$$


Proper substitution of equations (33) and (34) into equation (35) using equations (5), (6), (26), (27), (31), and (32) a long with equation (17) leads to the following expression for the maximum shear stress as a function of depth below a Hertzian contact, accounting for both ring press fit and ring speed.

$$
\tau=S_{\max }\left(t-u-\frac{1}{t}\right)-\left(m+A B^{2}\right)\left(\frac{1}{y^{2}}\right)+\frac{A}{2}(G-1) y^{2}-A B^{2}
$$

where, for simplicity

$$
\begin{aligned}
& m=\frac{P_{1} B^{2}}{1-B^{2}} \\
& A=K \omega^{2} r_{0}^{2}
\end{aligned}
$$

Again, taking the derivative of equation (47) with respect to $y$ and setting the result equal to zero leads to the value of $y$ where the shear stress is a maximum. Using the chain rule that $d s / d y-d s / d u \cdot d u / d y$,

$$
\frac{d \tau}{d y}=-S_{\max } K_{2}\left[\frac{u}{t}+\frac{u}{t^{3}}-1\right]+\frac{2\left(m+A B^{2}\right)}{y^{3}}+A(G-1) y=0
$$

Using the Lundberg-Palmgren (ref. 10) analys is where

$$
L=\left(\frac{k_{3} z_{0}^{h}}{\tau_{\max }^{c} v}\right)^{1 / e}
$$

where $Z_{0}$ is the depth to the maximum value of the maximum shearing stress from equation (49) and $\tau_{\max }$ is its value from equation (47), the effect of the press fits and centrifugal force on inner race iffe can be determined. The effects of press fit would not, however, affect the life-of the outer race. However, centrifugal loading of the rollers on the outer race does affect the outer race life which can be readily determined. Knowing the life of the inner and outer raceways, the $11 \mathrm{fe}$ of the bearing can be determined from LundbergPalmgren (ref. 10) as follows:

$$
\frac{1}{L^{e}}=\frac{1}{L_{i}^{e}}+\frac{1}{L_{0}^{e}}
$$

\section{RESULTS AND DISCUSSION}

\section{Inner-Race Life}

The relative values of subsurface stress are shown in figure 4 for the typical roller bearing inner-ring data given in table I. The principal stress in the $Z$-direction is shown in figure $4(a)$ while the principal stress in the 
$Y$-direction is shown in figure $4(b)$. The stress values have been nondimensionalized by the Hertz stress $\left(S_{\max }\right)$. Note that $y=0.90$ is the inner radfus and $y=1$ is the outer radius of the ring.

In figure $4(a)$, the stress due to ring speed is the largest component at the inner surface, but the subsurface compressive stress due to the Hertzian loading becomes dominant as the outer (contact) surface is approached. In figure $4(b)$, the stress due to ring speed is again the largest at the inner surface and the stress from the press fit is influential. Note that the ring speed is high in the example problem and that the pressure assumed for the press fit is probabiy moderate. However, the compressive stress due to the Hertzian loading becomes dominant as the outer surface is approached. The principal stress sy does remain tensile (positive) until about $y=0.995$, which will influence the maximum shear stress.

When the principal stresses from Fig. 4 are combined in accordance with equation (35) the result is the maximum shear stress, shown in figure 5 . To observe the change in the maximum shear stress when the effects of ring press fit and ring speed are added, one needs to compare the results in figure 5 with those due to Hertzian loading only (fig. 2). This is shown in figure 6, where the contributions to the maximum shear stress are shown separately. The increase in maximum value of the maximum shear stress for this example shown is about 33 percent. The solution to equation (49) for this example is

$y_{\max }=0.998204$, where $\tau_{\max }=-0.402 \mathrm{~S}_{\max }$. The depth to the maximum shear stress changed only very silghtly from that of the Hertz loading only.

The rolling-element fatigue life is taken to be inversely proportional to the maximum shear stress to the ninth power (ref. 10). Therefore, if we define a life ratio LR as the ratio of fatigue life based on the maximum shear stress that included the effects of ring speed and press fit to the fatigue life based on the maximum shear stress from the Hertzian loading only, we could write

$$
L R=\frac{L_{T}}{L_{H}}=\left[\frac{\tau_{\max , H}}{\tau_{\max , T}}\right]^{9}
$$

where $\tau_{\max }, \mathrm{H}$ is obtained from equation (20) and $\tau_{\max , T}$ is obtained from equation (47), using the value of $y$ obtained from equation (49). The value of $L R$, which is a measure of the effects of a press fit and ring speed, for the example is

$$
L R=\left(\frac{-0.30028 \times 1.4 \times 10^{9}}{-0.40167 \times 1.4 \times 10^{9}}\right)^{9}=0.0729
$$

That is, the corrected life is only 0.07 times that calculated using stresses from the Hertzian loading only.

To observe these effects over a broader range of variables, equations (47) and (49) were used to calculate the life ratio LR (eq. (52)) for ring thicknesses (B) from 0.5 to 0.92 , for Hertz stresses from $0.69 \times 10^{9}$ to $2.07 \times 10^{9} \mathrm{~N} / \mathrm{M}^{2}$ (100 000 to 300000 psi) using ring speeds of 500 and $2000 \mathrm{rad} / \mathrm{sec}$, for rings with an outer radius of 25.4 and $63.5 \mathrm{~mm}$ ( 1 and $2.5 \mathrm{in.}$ ), at a press fit pres- 
sure of $6.9 \times 10^{6} \mathrm{~N} / \mathrm{M}^{2}$ (1000 $\mathrm{psi}$ ), and with a radius ratio $R^{\prime}$ of both 5 and 10. The results are shown in figures 7 to 10 .

Figure 7, for a ring with an outer radius of $63.5 \mathrm{~mm}(2.5 \mathrm{in}$.) shows that the relative influence of press fit and ring speed on fatigue life diminishes as the Hertz stress is increased, or as the ring becomes thicker, or as the ring speed is decreased. Figure 8 shows the same data plotted against radius ratio. Several calculations for the conditions of figure 7 were made with $R^{\prime}=5$ and the 11 fe ratio results were the same. Increasing the press fit pressure would lower the values of LR. The pressure used in figures 7 and 8 represent an interference fit of about $0.051 \mathrm{~mm}(0.002 \mathrm{in.)}$ on the diameter.

Figures 9 and 10 show the results obtained for an outer radius of $25.4 \mathrm{~mm}$ ( 1.0 in.) and an $R^{\prime}$ of 5 . The life ratio results with an $R^{\prime}$ of 10 were the same. A comparison of figures 7 and 9 shows that the 1 ife ratios with the smaller outer radius were generally higher than for the corresponding larger ring, and were not so influenced by ring speed.

Since there are many values of LR of 0.5 or less, it may be concluded that the press fit and loading due to ring speed can have a significant effect on the fatigue life calculated for an inner-ring contact.

\section{Roller Bearing Life}

Using equations (50) and (51) the life of the entire bearing can be determined. These results are shown in nondimensional form in figure 11(a). For a bearing without any centrifugal force or press fit effects the normalized ilfe is one. Considering the effects of centrifugal force alone, the relative life at approximately $1.2 \mathrm{million}$ DN is approximately 60 percent the life using a straight Lundberg-Palmgren calculation of bearing catalog 1 ife. At the same speed, considering press fit alone, the life is also approximately 60 percent of the catalog calculation. However, both effects in combination produce a iffe of approximately 40 percent of the catalog calculation.

These effects at higher speeds are more dramatic. As an example, at approximately $3 \mathrm{milli}$ ion $\mathrm{DN}$, the effect of press $\mathrm{ftt}$ is lessened to produce a life of approximately 85 percent the catalog life. The effects of centrifugal force only produce a life of approximately 10 percent the catalog life. However, in combination, these effects can produce a life of only 8 or 9 percent the bearing catalog life.

\section{Effect of Mean Stress}

Lundberg-Palmgren (eq. (50)) in their original analysis considered only the maximum value of the reverse orthogonal shearing stress (ref. 10). Metallurgical studies of rolling-element specimens which failed in surface fatigue have shown, based upon crack propagation, that the critical shearing stresses can be the orthogonal, the octahedral or the maximum shear stress. All these shear stresses are a function of the maximum Hertzian (contact) stress. For the calculations performed herein the values of the maximum shear stress were used in equation (50) to determine bearing 1ife. For a given Hertzian stress and with the addition of hoop stresses due to press fits and centrifugal force, the mean value of the shear stress will increase. However, the amplitude of 
the maximum shear stress will remain unchanged. For a given operating condition, the hoop stresses impose a steady or static stress. The steady or static stress define the minimum shear stress. The maximum value of shear stress equals the amplitude of the maximum shear stress plus the static stress. The mean stress can be defined as the average of these two values.

A Goodman diagram type approach may be an effective but untried method to account for the effect of mean stress in rolling-element fatigue. Referring to figure 12, there is shown a proposed Goodman type diagram based upon the maximum shear stress amplitude $\tau_{a}$ and the mean shear stress, $\tau_{m}$. For the calculations of figure 12, the amplitude of the maximum shear stress $\tau_{a}$ on the bearing inner race was $0.39 \times 10^{9} \mathrm{~N} / \mathrm{M}^{2}$ (56 $100 \mathrm{ps} 1$ ). The mean value $\mathrm{T}_{\mathrm{m}}$ would be $0.19 \times 10^{9} \mathrm{~N} / \mathrm{M}^{2}$ (28 $050 \mathrm{psi}$ ). From reference 11 , the value of the fracture stress Su for AISI 52100 or AISI M-50 can be taken as $2.34 \times 10^{9} \mathrm{~N} / \mathrm{m}^{2}(340000 \mathrm{ps} 1)$. Based upon these values the effective maximum shear stress $\tau_{e}$ from figure 12 would be approximately $0.42 \times 10^{9} \mathrm{~N} / \mathrm{M}^{2}$ (61 $144 \mathrm{ps} 1$ ) based upon the following proposed equation for a Goodman diagram.

$$
\tau_{e}=\frac{\tau_{a} \cdot \tau_{m}}{S_{u}-\tau_{m}}+\tau a=\frac{\tau_{a}}{1-\left(\frac{\tau_{m}}{S_{u}}\right)}
$$

The values of $\tau_{e}$ from equation (53) can be used in equations (50) and (51) as the va le of $\tau_{\max }$ to predict bearing life. This was done for the data of figure $11(a)$, the results of which are shown in figure $11(b)$. The results of the analysis indicated bearing lives for the conditions shown ranging from 70 to 80 percent of those 11 ves where there are no effects of hoop stresses due to centrifugal force and press fits. These lives contrast with those predictions shown for comparison in figure 11(b) based upon only considering the maximum shear stress $\tau_{\max }$ as a result of hoop stress without considering the effect of mean shear stress. It becomes apparent that the reduction in lives predicted using a value of $\tau_{e}$ from a Goodman diagram which considers hoop stresses is less significant than using the resultant value of the maximum shear stress, $\tau_{\max }$. Experimental research needs to be performed to verify and refine this approach.

\section{SUMMARY}

An analysis was performed to determine the effects of inner-ring speed and press fits on roller bearing fatigue life. The effects of the resultant hoop and radial stress on the principal stresses were considered. The maximum shear stresses below the Hertzian contact were determined for different conditions of inner-ring speed and load and were applied to a conventional roller bearing ilfe analysis. The effect of mean stress was determined using a Goodman diagram approach. The following results were obtained.

1. Hoop stresses caused by press fits and centrifugal force can reduce bearing 11 fe by as much as 90 percent.

2. Use of a Goodman diagram calculation considering mean maximum shear stress and shear stress amplitude to determine an effective maximum shear stress resulted in the calculation of roller bearing life 20 to 30 percent less than theoretical life predictions. 
3. The depth to the maximum shear stress remains relatively unchanged by speed and press fit, over the range calculated, from that estabilshed by conventional theory.

\section{REFERENCES}

1. Scott, R.L.; Kepple, R.K., and Miller, M.H., "The Effect of Processinginduced Near-surface Residual Stress on Ball Bearing Fatigue," Rolling Contact Phenomena, J.B. Bidwe11, Ed., Elsevier, 1962, pp. 301-316.

2. Zaretsky, E.V., Parker, R.J., Anderson, W.J., and Miller, S.T., "Effect of Component Differential Hardnesses on Residual Stress and Rolling-Contact Fatigue," NASA TN-D-2664, 1965.

3. Alman, J.0.: Effects of Residual Stress on Rolling Bodies. Rolling Contact Phenomena, J.B. Bidwell, Ed. Elsevier, 1962, pp. 400-424.

4. Townsend, D.P., and Zaretsky, E.V., "Effect of Shot Peening on Surface Fatigue Life of Carburized and Hardened AISI 9310 Spur Gears," NASA TP-2047, Aug. 1982.

5. Czyzewski, T., "Influence of a Tension Stress Field Introduced in the Elastohydrodynamic Contact Zone on Rolling-Contact Fatigue," Wear. Vo1. 34, pp. 201-214, 1975.

6. Harris, T.A., Rolling Bearing Analyses, John Wiley and Sons, Second Edition, NY, 1984 .

7. Jones, A.B., "Analysis of Stress and Deflections," Vol. I, New Departure Div., General Motors Corp, 1946.

8. Thomas, H.R., and Hoersch, V.A., Stresses due to the Pressure of One Elastic Solid on Another, "Bulletin of Engineering Experiment Station No. 212, University of Illinois, 1930.

9. Faupe1, J.H.: Engineering Design. John Wiley and Sons, Inc. 1964.

10. Lundberg, G.; and Palmgren, A., "Dynamic Capacity of Rolling Bearings," Acta Polytech. Scand, Mech. Engr. Ser., Vol. 1, no. 3, 1947.

11. Sachs, G., Se11, R., and Brown, W.F., Jr., "Tension, Compression, and Fatigue Properties of Several Steels for Aircraft Bearing Applications, Trans. ASTM, 1959, pp. 1-23. 
TABLE I. - VALUES FOR EXAMPLE PROBLEM

\begin{tabular}{|l|l|}
\hline \multicolumn{2}{|c|}{ Variables } \\
\hline$B$ & 0.90 \\
$P_{1}$ & $6.89 \times 10^{6} \mathrm{~N} / \mathrm{M}^{2}(1000 \mathrm{psi})$ \\
$\omega$ & $2000 \mathrm{rad} / \mathrm{sec}$ \\
$r_{0}$ & $63.5 \mathrm{~mm}(2.5 \mathrm{in.})$ \\
$R^{\prime}$ & 10 \\
$S_{\text {max }}$ & $1.379 \times 10^{9} \mathrm{~N} / \mathrm{M}^{2}(200000 \mathrm{psi})$ \\
\hline & \multicolumn{1}{|c|}{$\operatorname{Constants}^{2}$} \\
\hline$K$ for steel & $3352 \mathrm{~N}-\sec ^{2} / \mathrm{M}^{4}\left(3.14 \times 10^{-4} \mathrm{lb}-\mathrm{sec}^{2} / 1 \mathrm{n} .{ }^{4}\right)$ \\
$G$ & 0.1667 \\
$E^{\prime}$ & $9.11 \times 10^{-12} \mathrm{M}^{2} / \mathrm{N}\left(6.28 \times 10^{-8} \mathrm{in.}^{2} / 1 \mathrm{~b}\right)$ \\
\hline
\end{tabular}



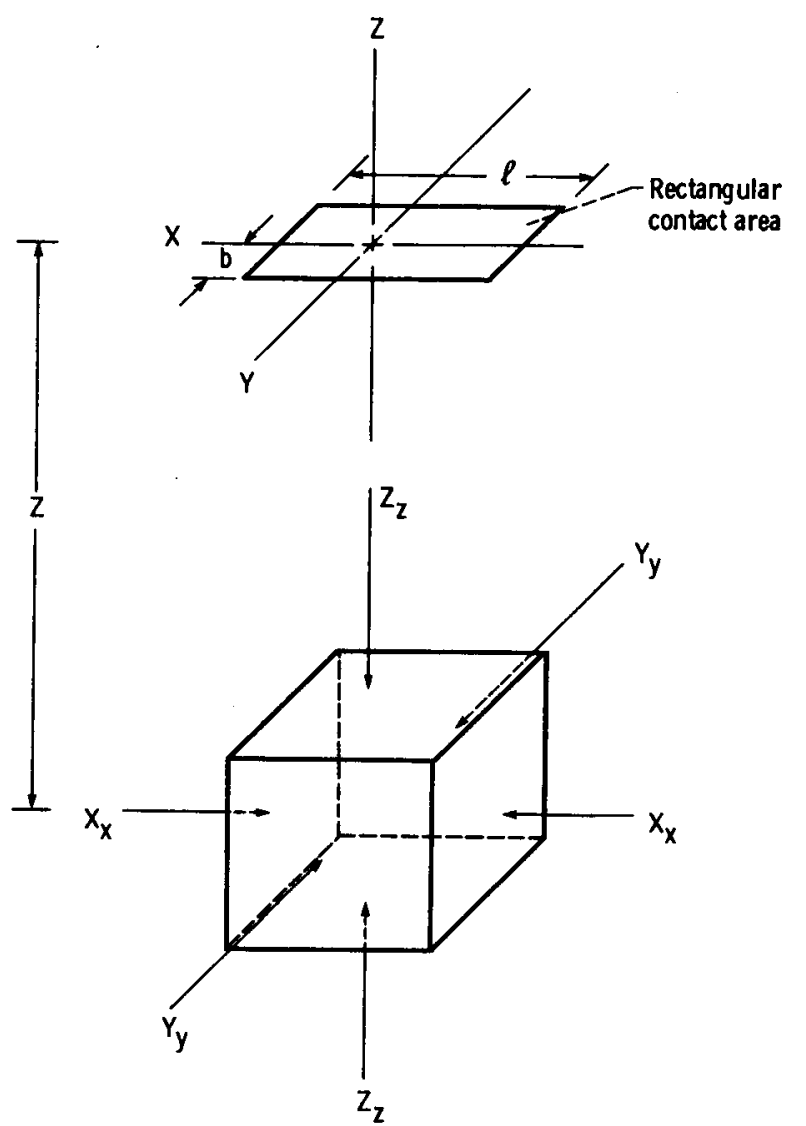

Figure 1. - Principal stresses on elementary subsurface particle at depth $Z$ under Hertzian pressure area. 


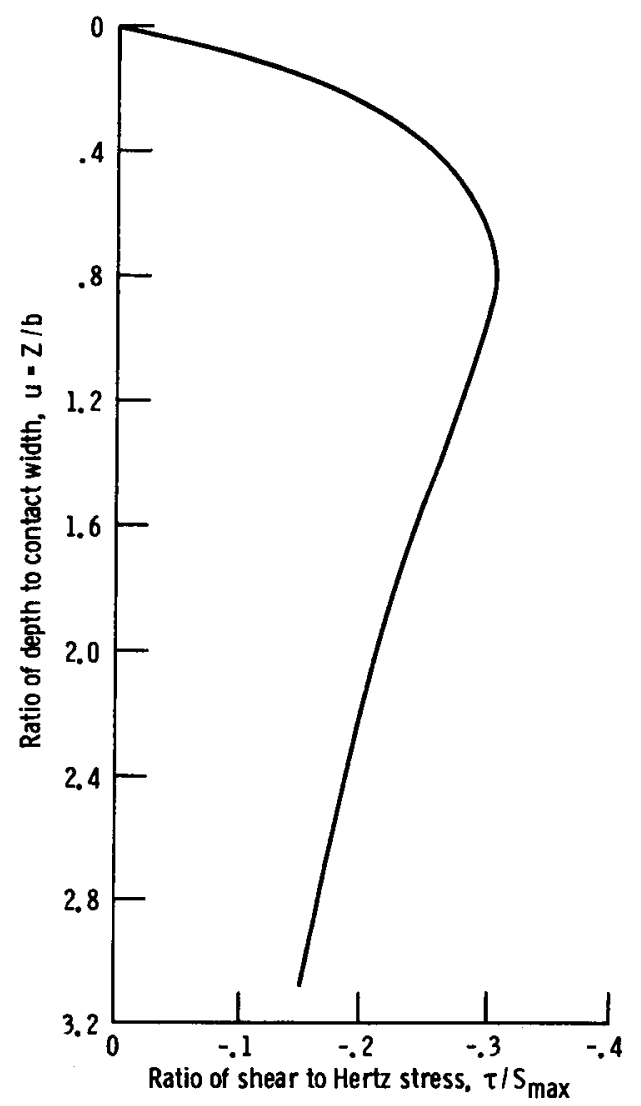

Figure 2 - Maximum shear stress as a function of depth below the surface, for Hertzian loading. Stress on Z-axis, below rectangular pressure area. 


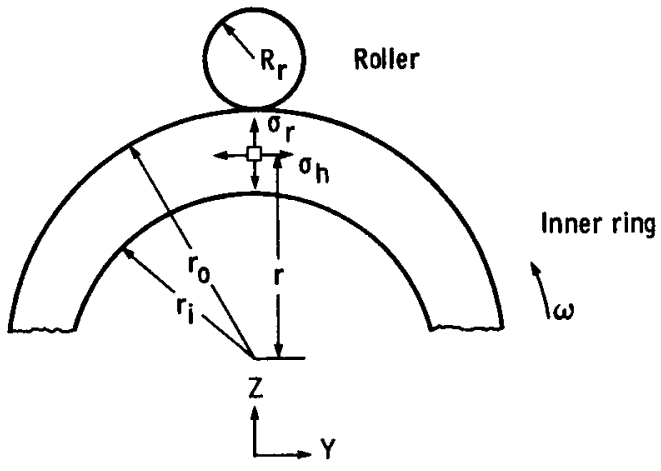

Fiqure 3. - Definition of additional terms used in analysis of subsurface stress in roller bearing inner ring.

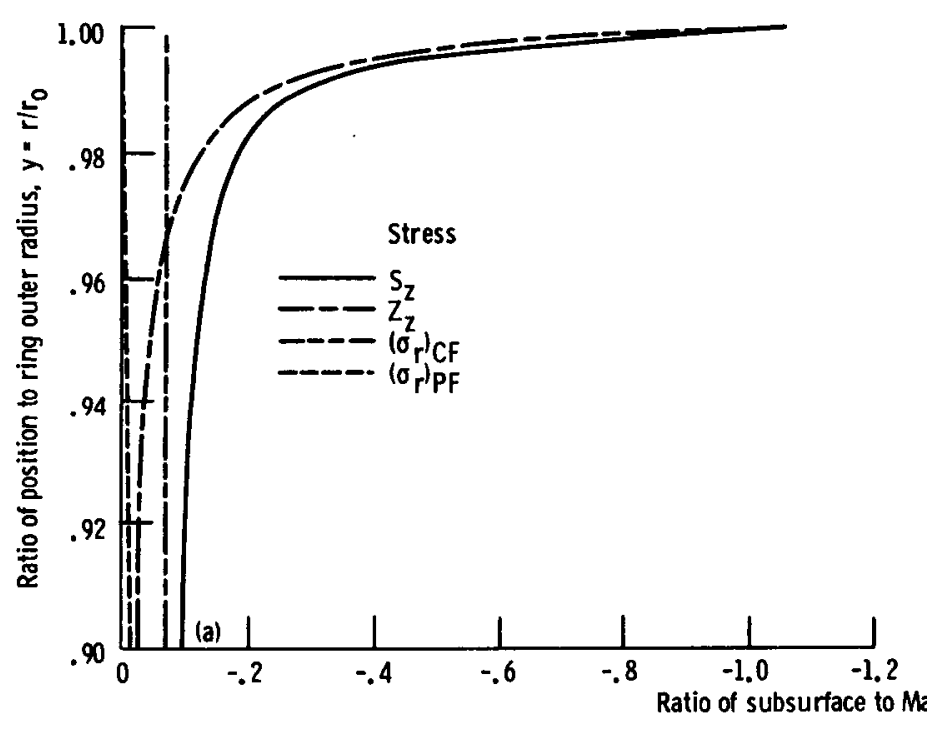

(a) In Radial, or Z-direction.

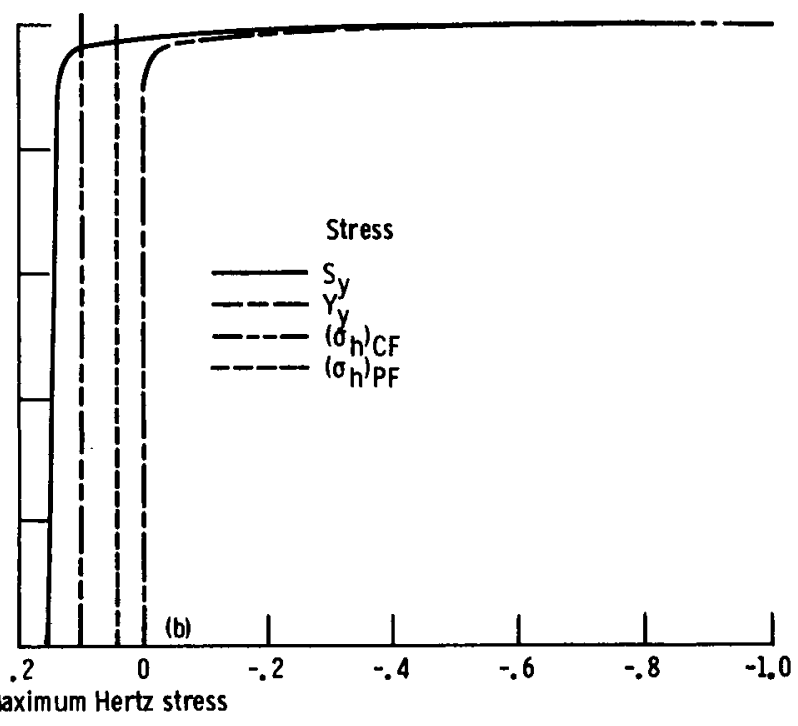

(b) Hoop, or Y-direction.

Figure 4 - Components of subsurlace stress in roller bearing inner ring, for conditions described in Table $I$. 


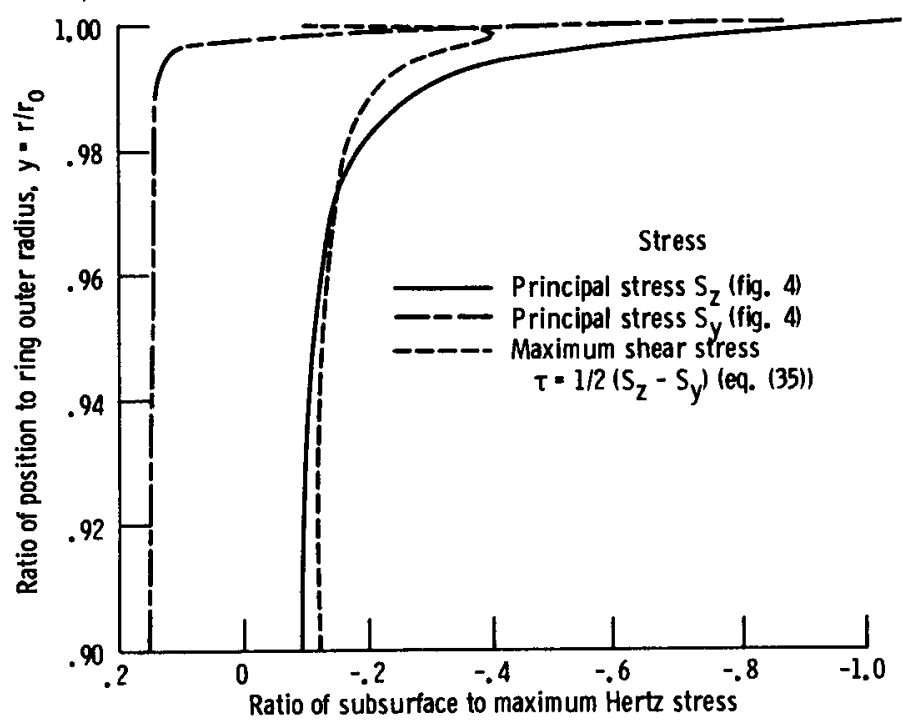

Figure 5. -Principal and maximum shear stresses in roller bearing inner ring. for conditions described in Table I.

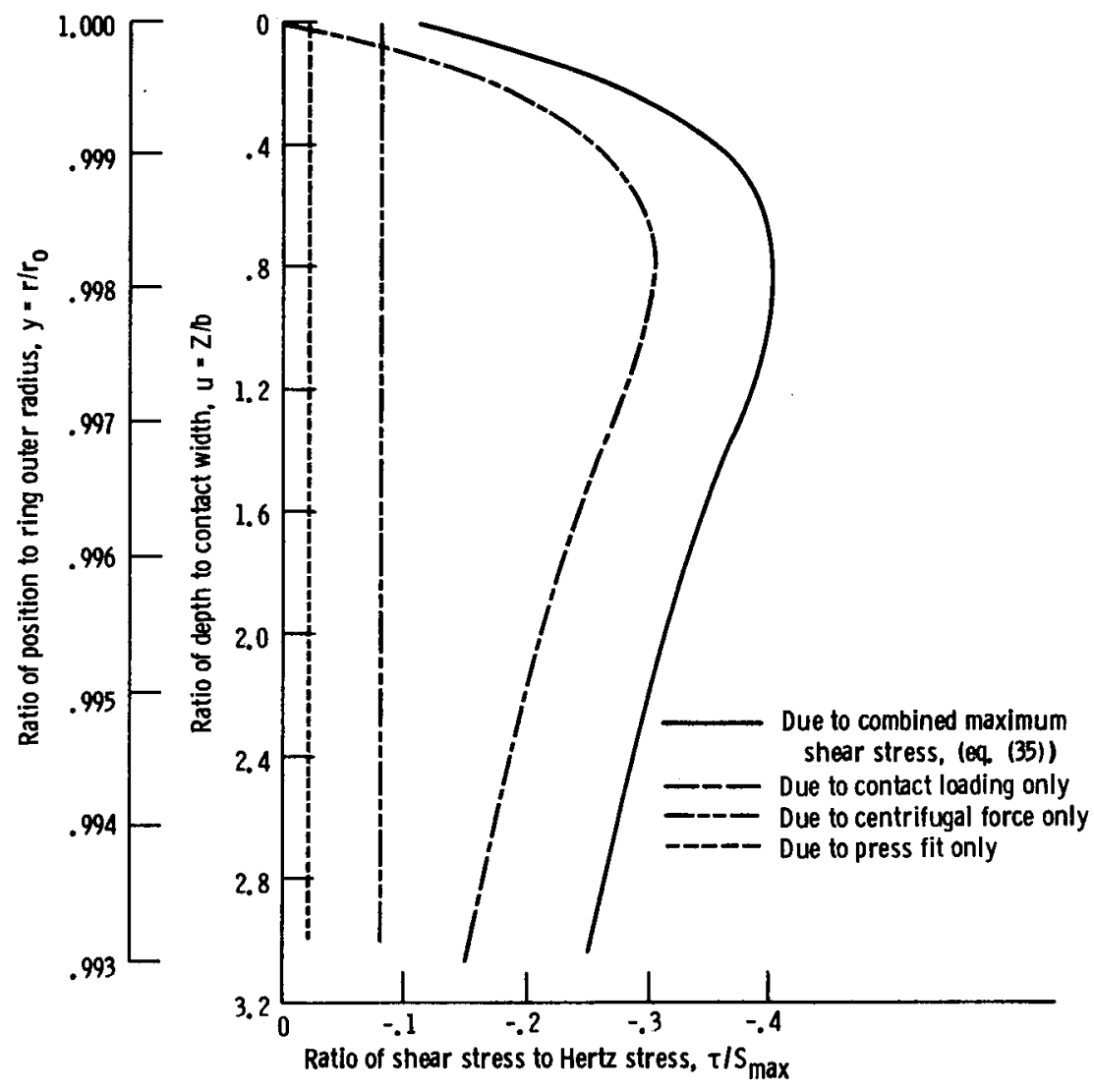

Figure 6. - Components of combined maximum shear stress in roller bearing inner ring, for conditions described in Table I 


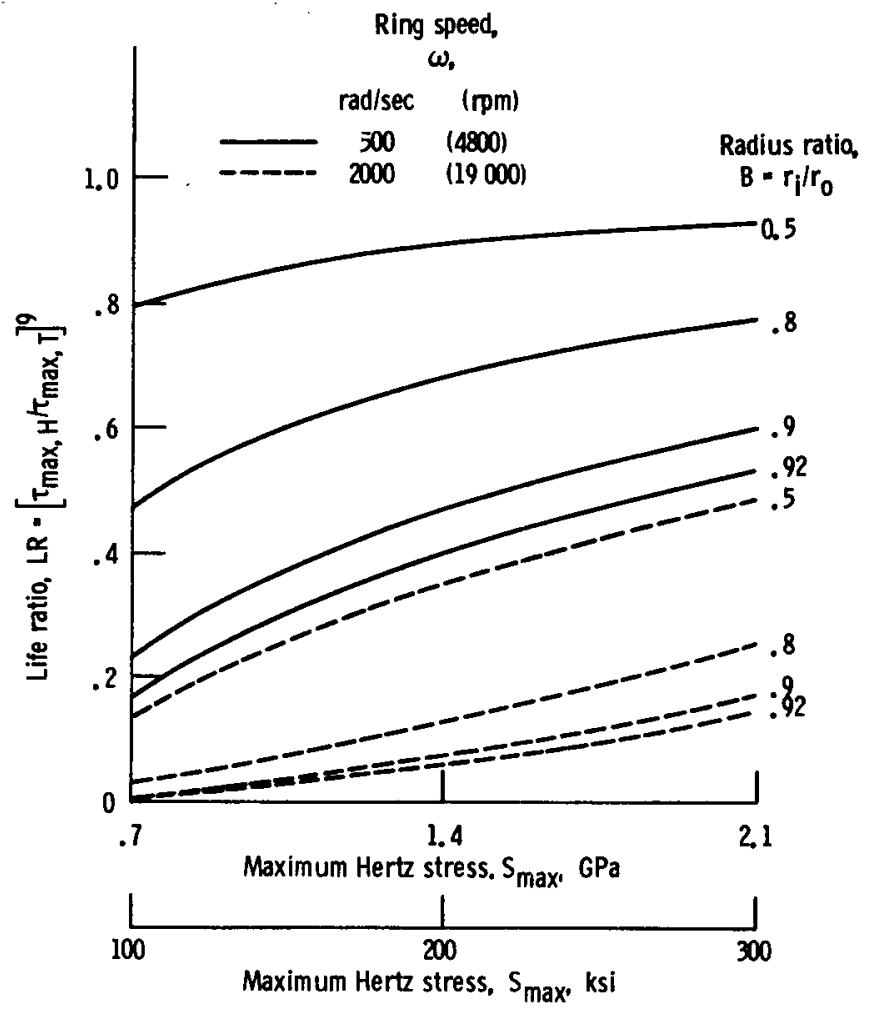

Figure 7. - Life ratio as a function of Hertz stress, for several ring thicknesses. at two ring speeds. $r_{0}=63.5 \mathrm{~mm}$ (2.5 in. ), $P_{j}=6.0 \times 10^{6} \mathrm{~N} / \mathrm{m}^{2}(1000 \mathrm{psi}) \mathrm{R}^{\prime}=10$

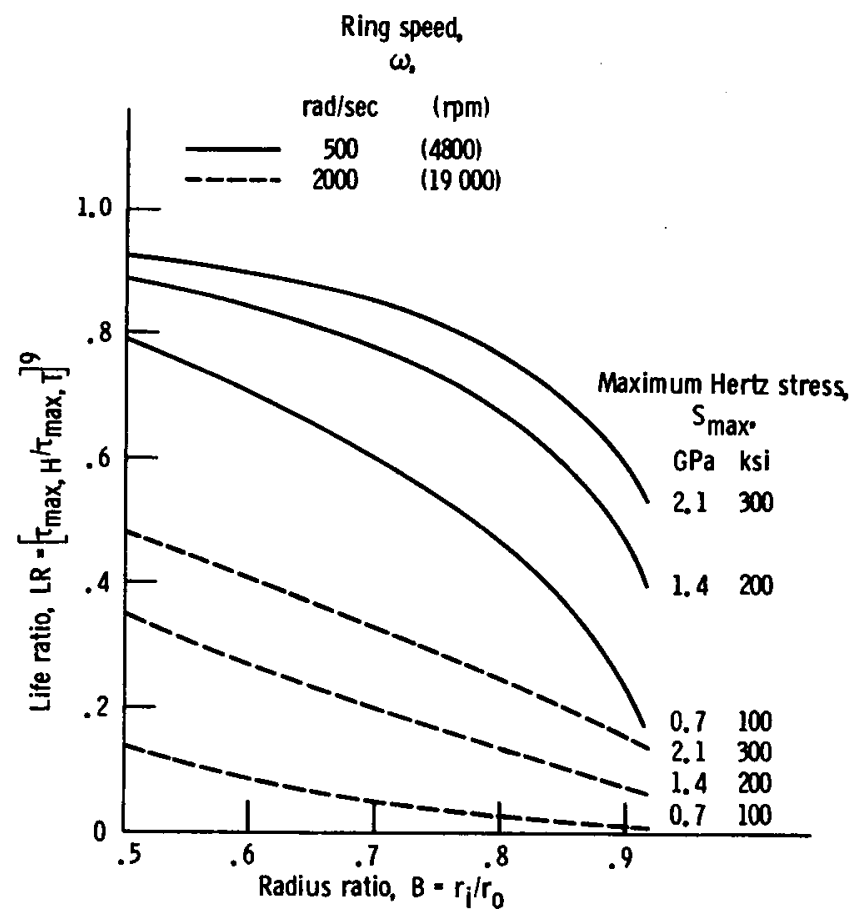

Figure 8 - Life ratio as a function of radius ratio for three values of maximum Hert stress, at two ring speeds. $r_{0}=63.5 \mathrm{~mm}$ (25 in. ), $P_{i}=6.9 \times 10^{6} \mathrm{~N} / \mathrm{m}^{2}$ (1000 psi), $R^{\prime}=10$. 


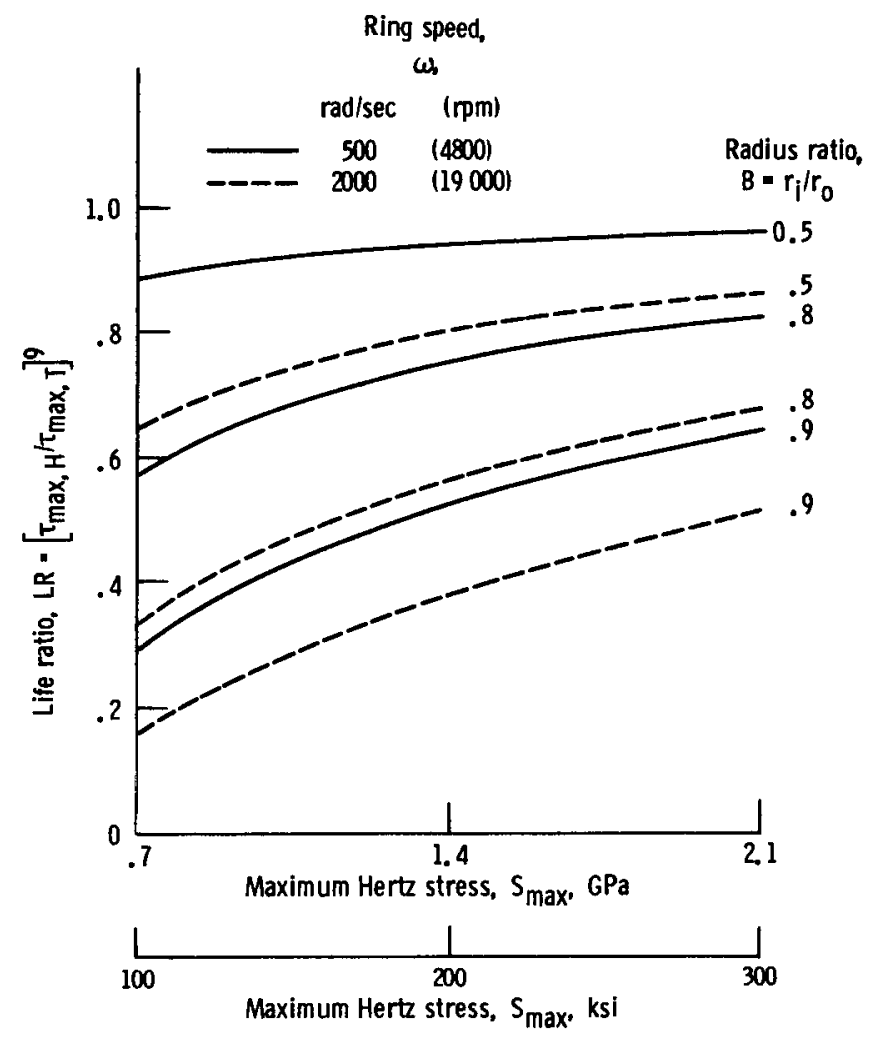

Fiqure 9. - Life ratio as a function of Hertz stress, for three ring thicknesses and two ring speeds. $r_{0}=25.4 \mathrm{~mm}$ (1. 0 in.), $P_{i}=6.9 \times 10^{6} \mathrm{~N}\left(\mathrm{~m}^{2}(1000 \mathrm{psi}), R^{\prime}=5\right.$.

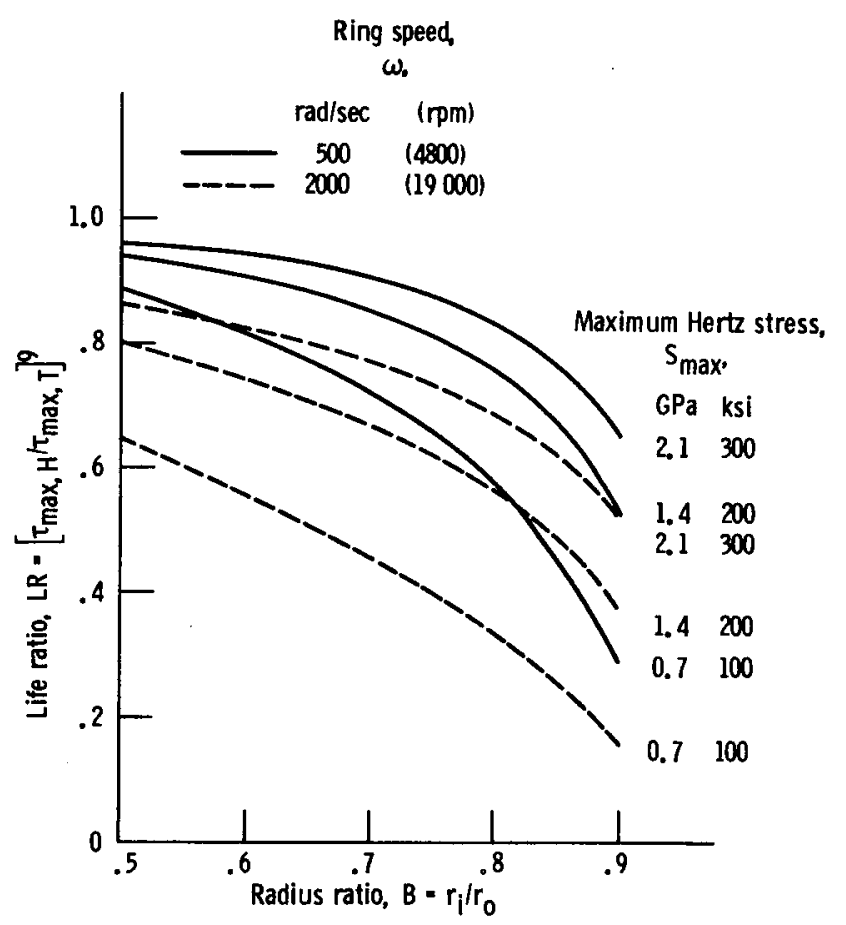

Fiqure 10. - Life ratio as a function of radius ratio for three values of Hertz stress, at two ring speeds. $r_{0}=25.4 \mathrm{~mm}$ (1. 0 in. ), $P_{i}=6.9 \times 10^{6} \mathrm{~N} / \mathrm{m}^{2}(1000 \mathrm{psi}), R^{\prime}=\xi$. 


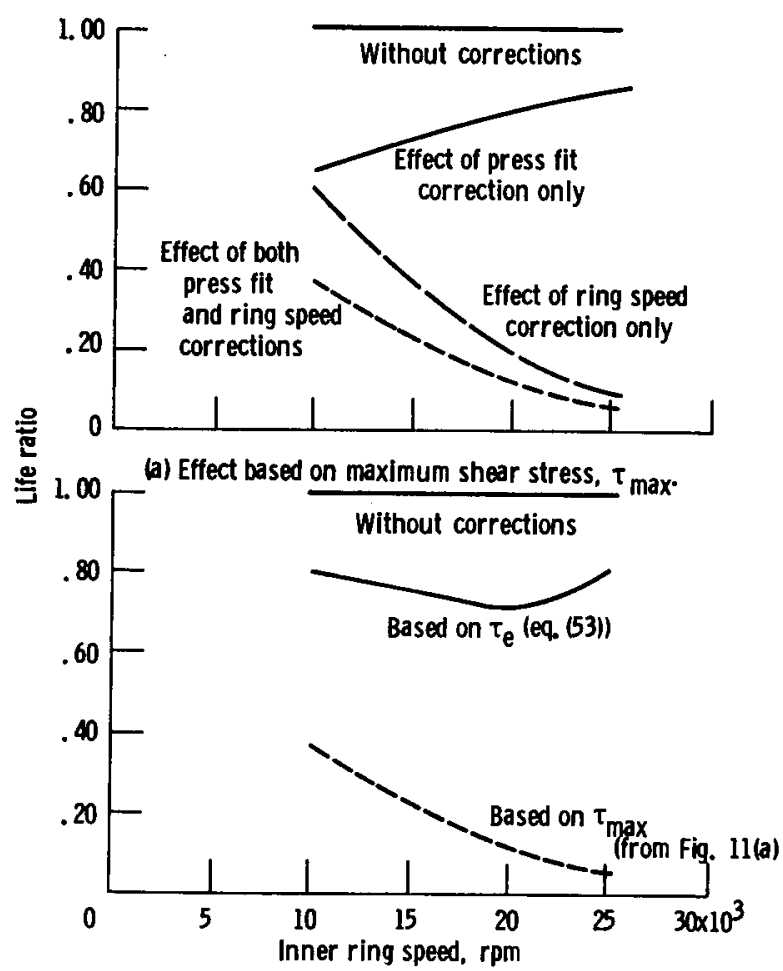

(b) Effect of both press fit and ring speed life corrections based on $\tau_{\mathrm{e}}$ and $\tau_{\max }$.

Figure 1L - Effect of hoop stresses on the fatigue life of $118-\mathrm{mm}$ bore roller bearing. Radial load $8900 \mathrm{~N}(2000 \mathrm{lb})$. Interference fit pressure $6.89 \times 10^{6} \mathrm{~N} / \mathrm{m}^{2}(1000 \mathrm{psi})$.

Maximum Hertz stress at maximum loaded roller 1290 $\mathrm{N} / \mathrm{m}^{2}(187000 \mathrm{psi})$.

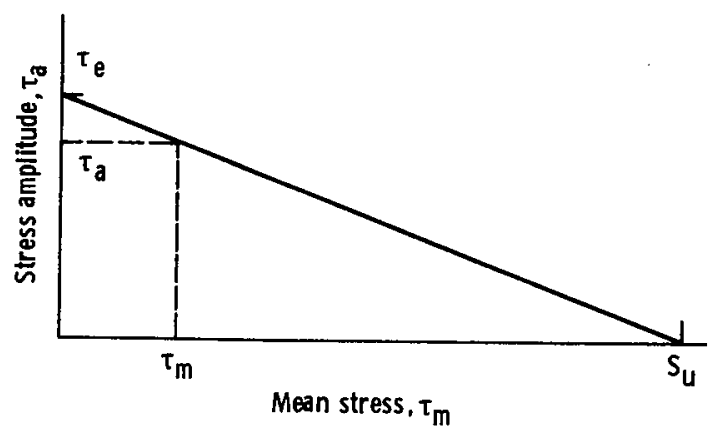

Figure 12 - Proposed Goodman diagram for rolling-element fatigue life predictions to account for effects of hoop stresses. 


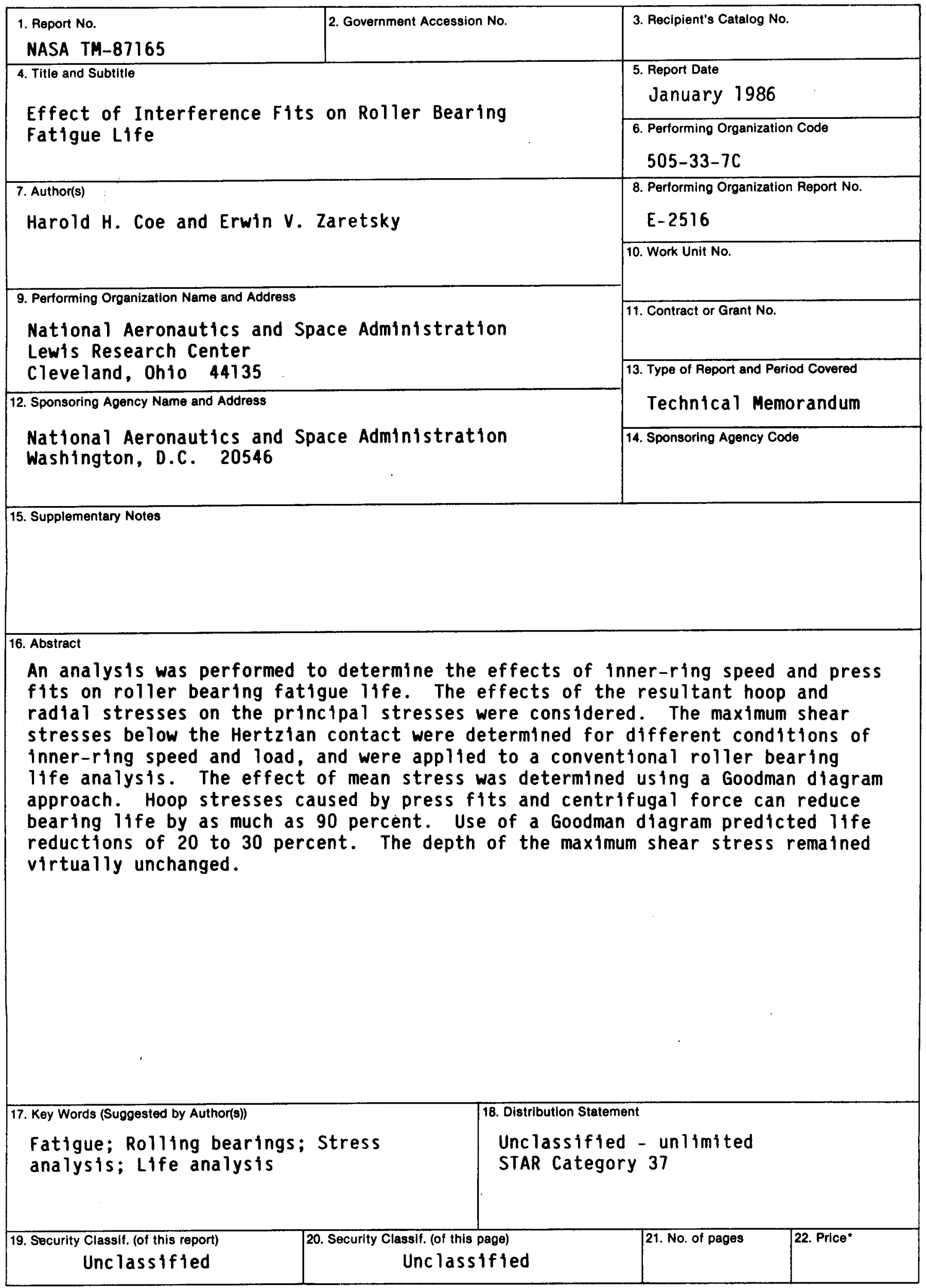

"For sale by the National Technical Information Service, Springfield, Virginia 22161 